Supporting Information for:

Kinetically Dependent Self-Assembly of Chiral Block Copolymers under 3D Confinement

Hao Li ${ }^{1}$, Xi Mao ${ }^{l}$, Huayang Wang ${ }^{1}$, Zhen Geng ${ }^{l}$, Bijin Xiong ${ }^{l}$, Lianbin Zhangl, Simin Liu ${ }^{2}$, Jiangping $X u^{l,}{ }^{*}$ and Jintao Zhu ${ }^{l, *}$

${ }^{1}$ State Key Lab of Materials Processing and Die \& Mould Technology and Key Lab of Materials Chemistry for Energy Conversion \& Storage (HUST) of Ministry of Education, School of Chemistry and Chemical Engineering, Huazhong University of Science and Technology (HUST), Wuhan 430074, China

${ }^{2}$ School of Chemistry and Chemical Engineering, Wuhan University of Science and Technology, Wuhan 430081, China

*Corresponding authors: E-mail: jiangpingxu@hust.edu.cn (J. X.);

jtzhu@mail.hust.edu.cn (J. Z.) 


\section{Supporting Scheme:}

(a)

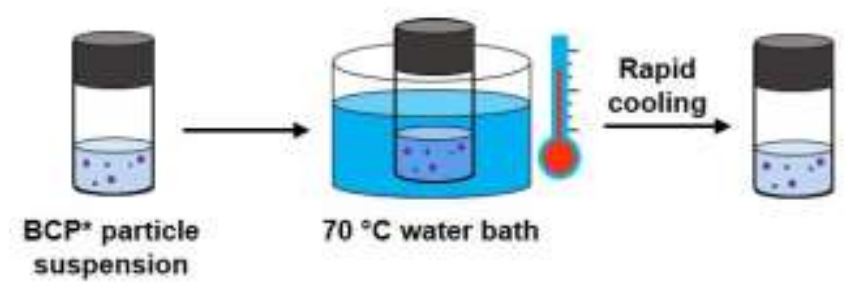

(b)

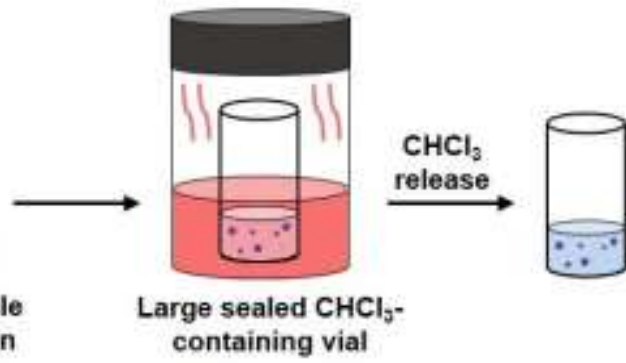

Scheme S1. Schematic illustrations showing the thermal annealing (a) and solvent-absorption annealing (b) of $\mathrm{BCP}^{*}$ particles. Prior to annealing, the pristine $\mathrm{PS}_{21 \mathrm{k}}-b$-PDLA $14 \mathrm{k} \mathrm{BCP}^{*}$ particles were diluted to $0.05 \mathrm{mg} / \mathrm{mL}$ in CTAB aqueous solution (3 $\mathrm{mg} / \mathrm{mL}$ ). Then, $0.2 \mathrm{~mL}$ of the particle suspension was transferred into a $2 \mathrm{~mL}$ glass vial.

For thermal annealing (a), the vial was sealed and then incubated in $70{ }^{\circ} \mathrm{C}$ water bath for a desired time. After annealing, the $2 \mathrm{~mL}$ vial was rapidly cooled to room temperature.

For solvent-absorption annealing (b), the $2 \mathrm{~mL}$ vial was kept open and placed in a 20 $\mathrm{mL}$ glass vial, which contained $2 \mathrm{~mL}$ of $\mathrm{CHCl}_{3}$. Next, the $20 \mathrm{~mL}$ vial was tightly sealed and stood for a desired time at $30^{\circ} \mathrm{C}$. After annealing, the $20 \mathrm{~mL}$ vial was removed and the $2 \mathrm{~mL}$ vial was kept in open air for $12 \mathrm{~h}$ to release the absorbed $\mathrm{CHCl}_{3}$. 


\section{Supporting Figures:}
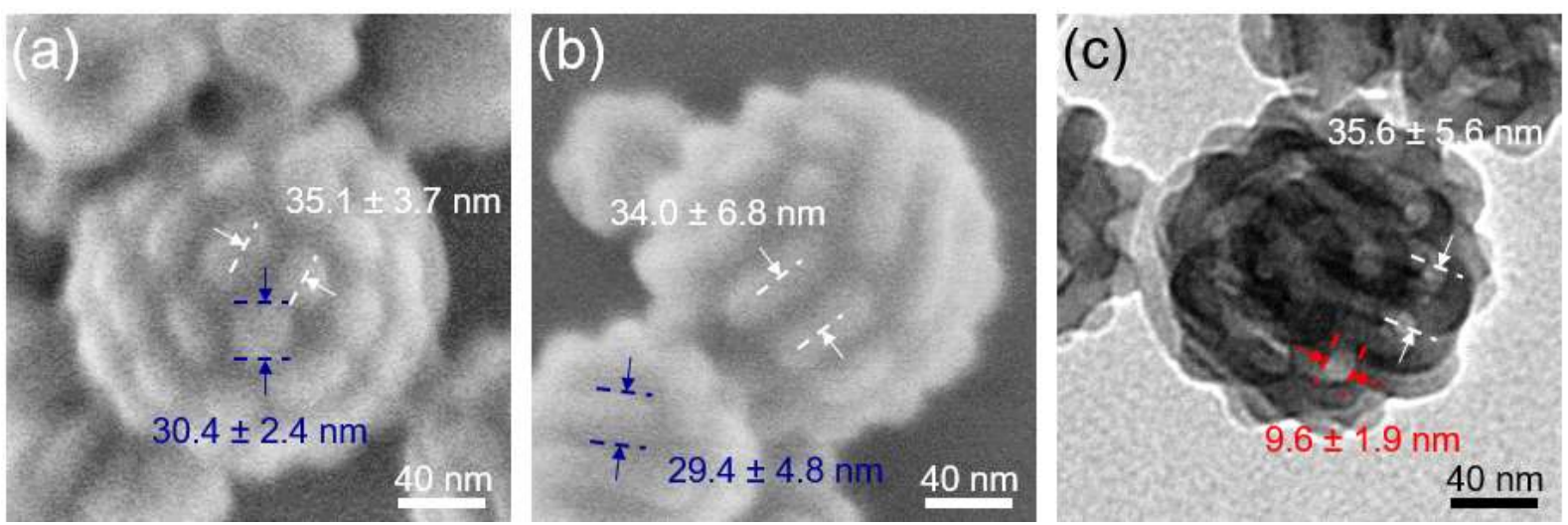

Figure S1. High magnification EM images of morphology I particles from PS $21 k-b$-PDLA $14 k$ BCP* $^{*}$ (a) SEM image of the dotted surface pattern. The diameter of dot protuberances is $30.4 \pm 2.4 \mathrm{~nm}$ (indicated by dashed blue lines), and the domain spacing is $35.1 \pm 3.7 \mathrm{~nm}$ (indicated by dashed white lines). (b) SEM image of the randomly striped surface pattern. The width of short stripe protuberances is $29.4 \pm 4.8 \mathrm{~nm}$ ("groove to groove", indicated by dashed blue lines), and the domain spacing is $34.0 \pm 6.8 \mathrm{~nm}$ ("ridge to ridge", indicated by dashed white lines). (c) TEM image of the internal coil-like helical structure. The diameter of helical domains is $9.6 \pm 1.9 \mathrm{~nm}$ (indicated by dashed red lines), and the pitch length is $35.6 \pm$ $5.6 \mathrm{~nm}$ (indicated by dashed white lines). The dimensions were determined by measuring over 200 particles with the software of ImageJ. 

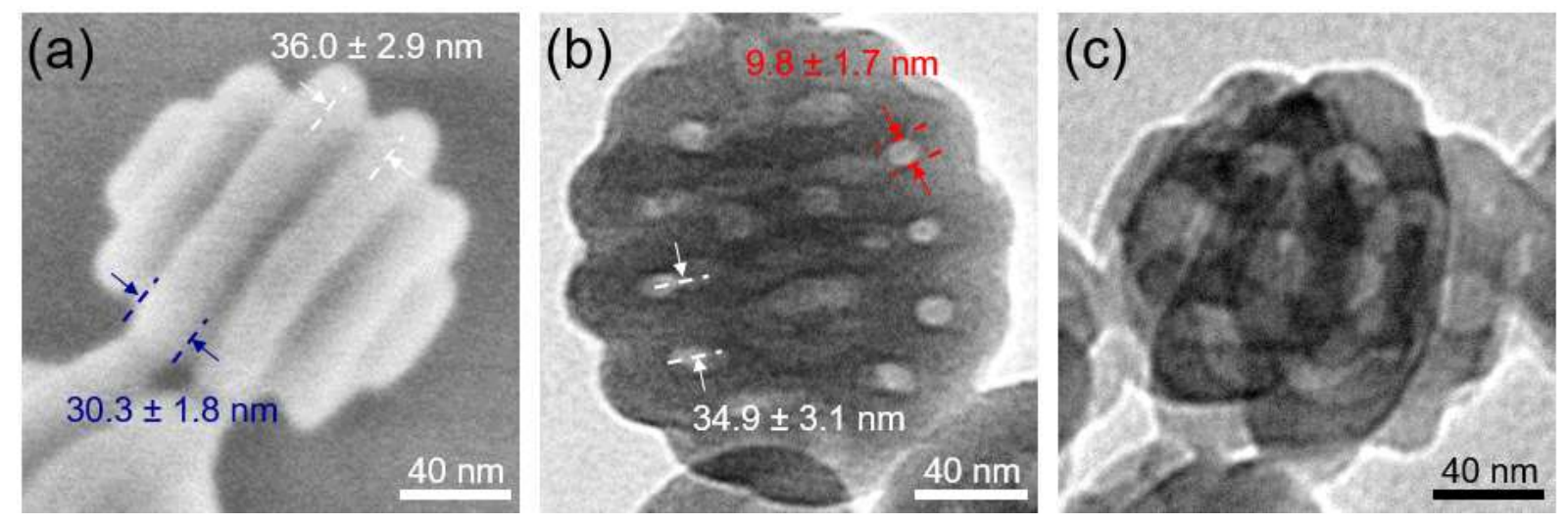

Figure S2. High magnification EM images of morphology II particles prepared from $P S_{21 k}-b-P D L A_{14 k} B C P^{*}$. (a) SEM image of the parallelly striped surface pattern. The width of stripe flanges is $30.3 \pm 1.8 \mathrm{~nm}$ ("groove to groove", indicated by dashed blue lines), while the domain spacing is $36.0 \pm 2.9 \mathrm{~nm}$ ("ridge to ridge", indicated by dashed white lines). (b-c) TEM images of the internal loop-like cylindrical structure. The diameter of cylindrical domains is $9.8 \pm 1.7 \mathrm{~nm}$ (indicated by dashed red lines), and the domain spacing is $34.9 \pm$ $3.1 \mathrm{~nm}$ (indicated by dashed white lines). The dimensions were determined by measuring over 200 particles with the software of ImageJ. 


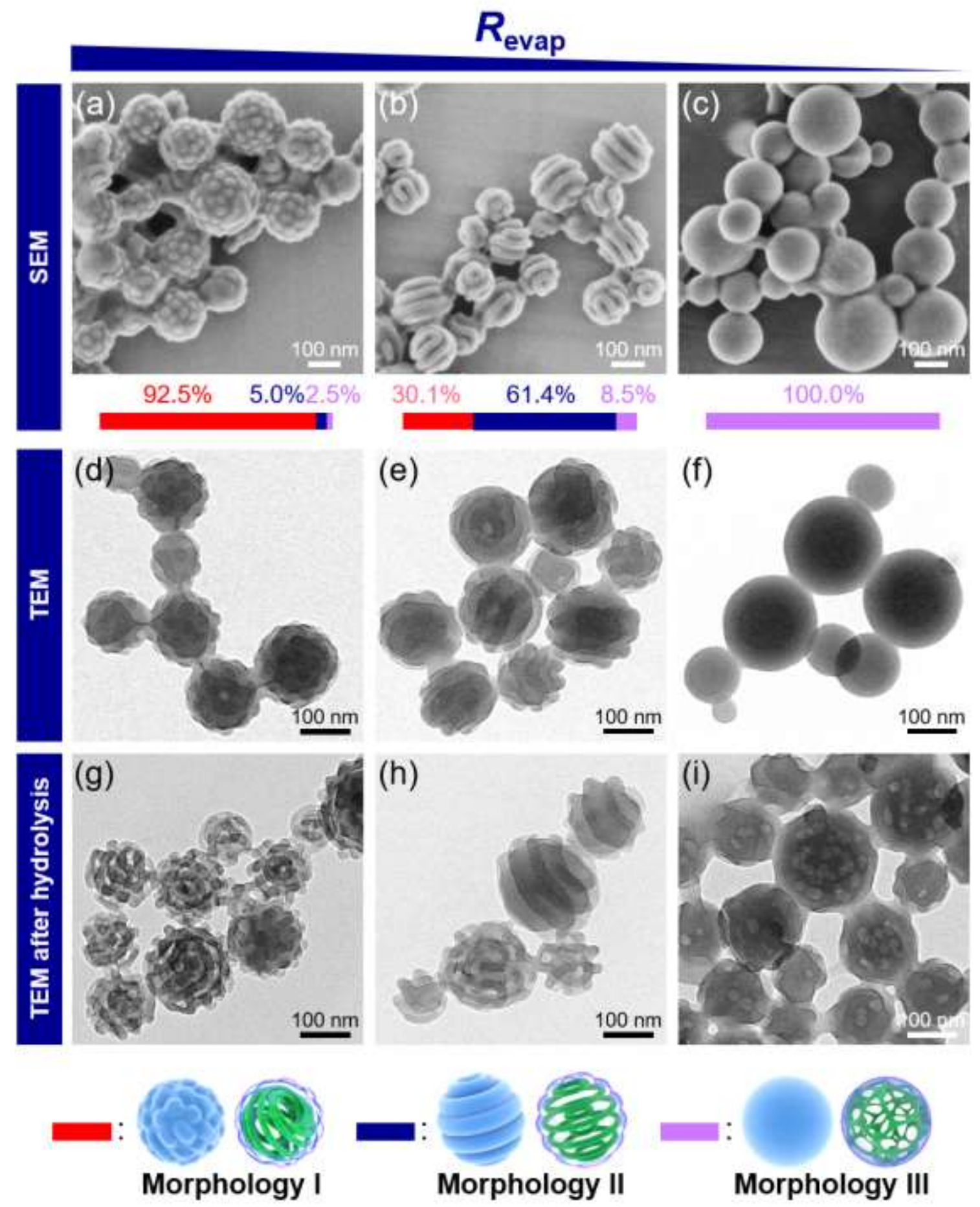

Figure S3. SEM (a-c) and TEM (d-f) images of PS $21 k-b-P D L A_{17 k}$ particles prepared at

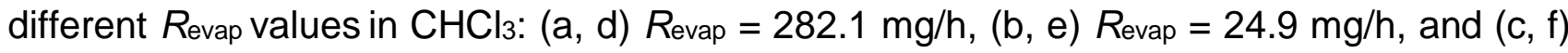
$R_{\text {evap }}=15.3 \mathrm{mg} / \mathrm{h}$. (g-i) TEM images of hydrolyzed particles corresponding to (d) $-(\mathrm{f})$, respectively. Bar charts present the frequency of each morphology, I (red), II (navy blue) and III (purple), by counting 300-600 particles under each condition. 


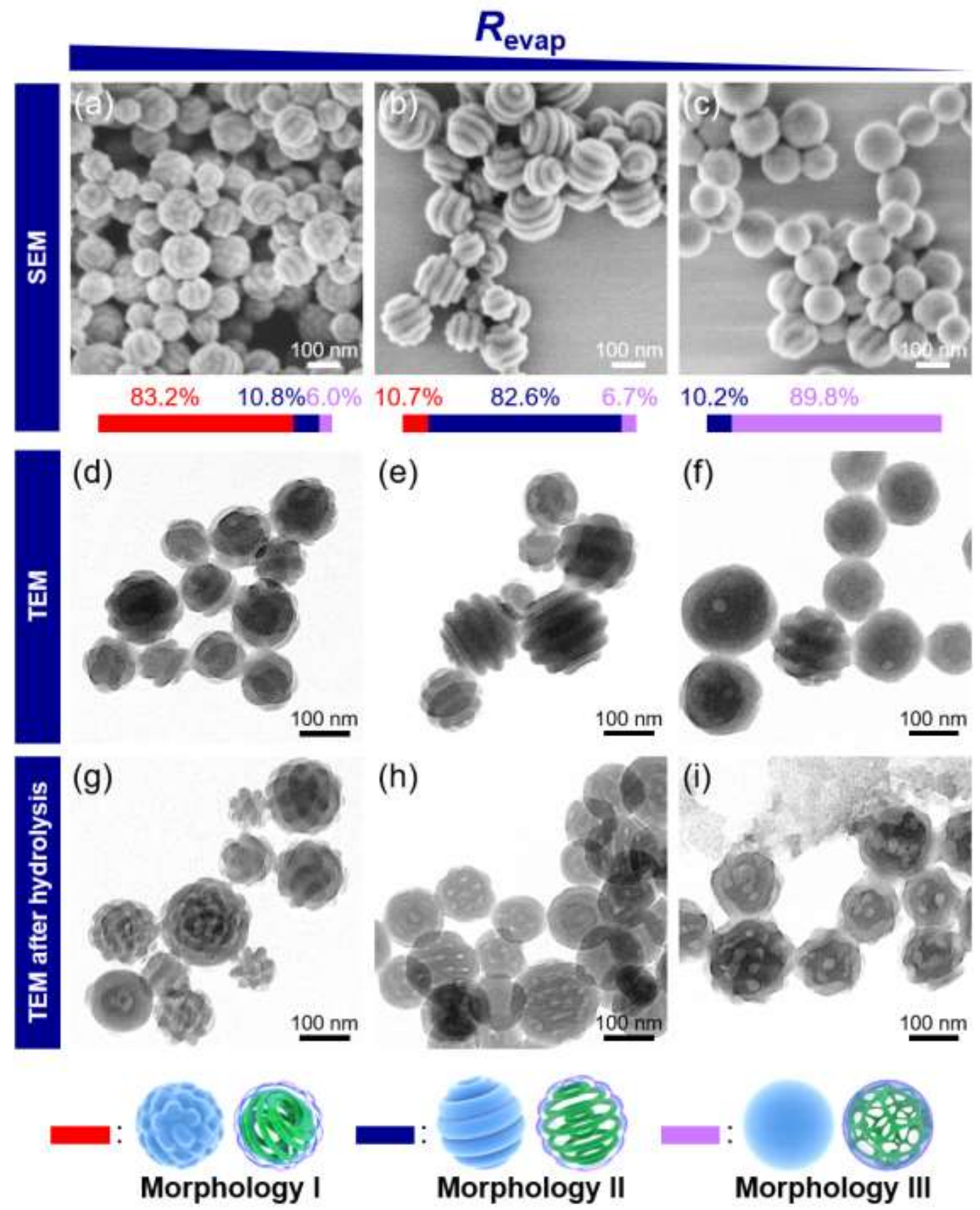

Figure S4. SEM (a-c) and TEM (d-f) images of PS ${ }_{21 k}-b-P D L A_{14 k}$ particles prepared at different $R$ evap values in $\mathrm{C}_{2} \mathrm{H}_{4} \mathrm{Cl}_{2}$ : (a, d) condition I, (b, e) condition II, and (c, f) condition III. ( $g-i)$ TEM images of hydrolyzed particles corresponding to (d)-(f), respectively. Bar charts present the frequency of each morphology, I (red), II (navy blue) and III (purple), by counting 300-600 particles under each condition. 


\section{$\boldsymbol{R}_{\text {evap }}$}
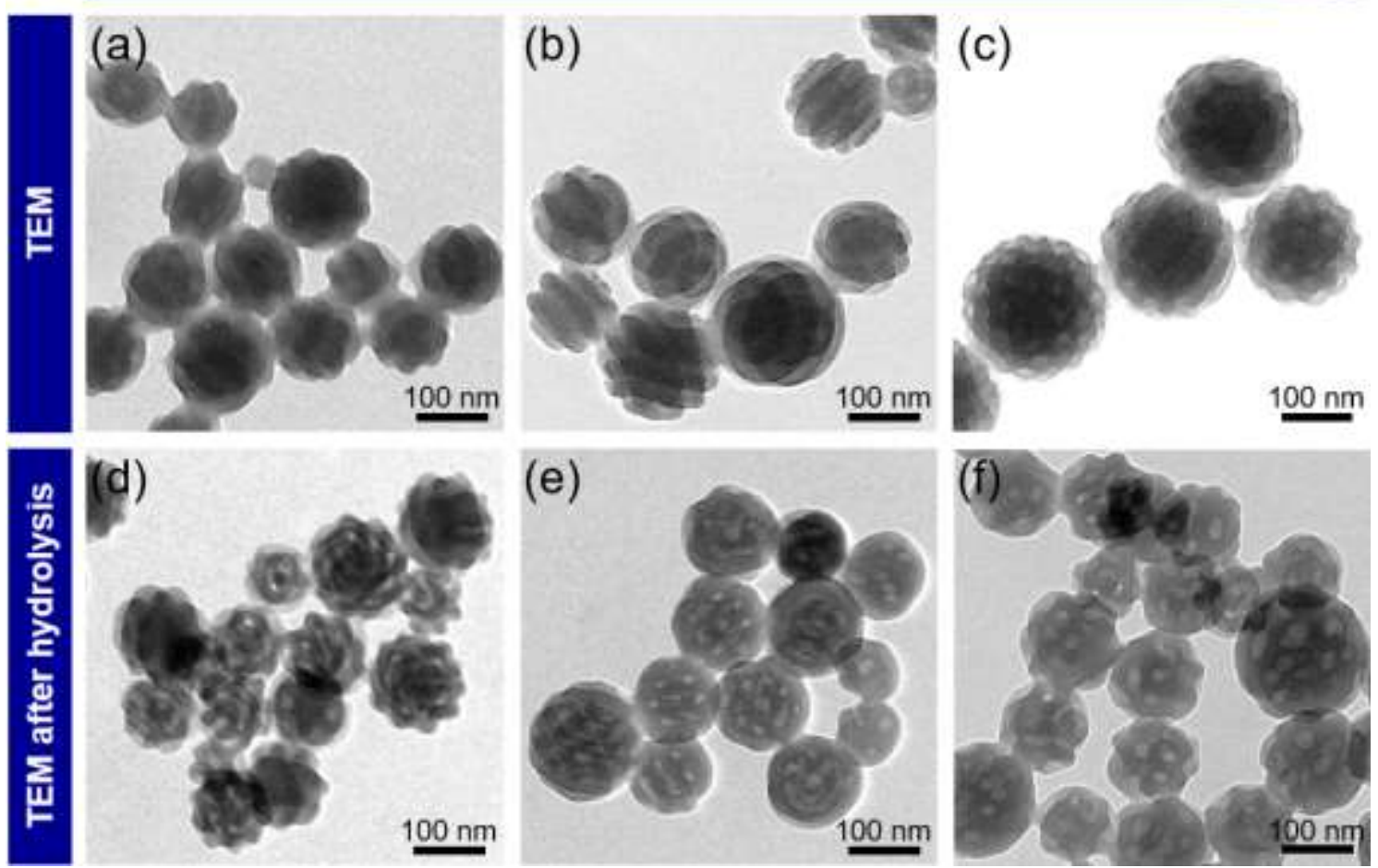

Figure S5. (a-c) TEM images of PS $21 \mathrm{k}-b-\mathrm{PDLA}{ }_{14 \mathrm{k}}$ particles prepared at different $R_{\text {evap values }}$ in $\mathrm{CH}_{2} \mathrm{Cl}_{2}$ : (a) condition I, (b) condition II, and (c) condition III. (d-f) TEM images of hydrolyzed particles corresponding to (a)-(c), respectively. 


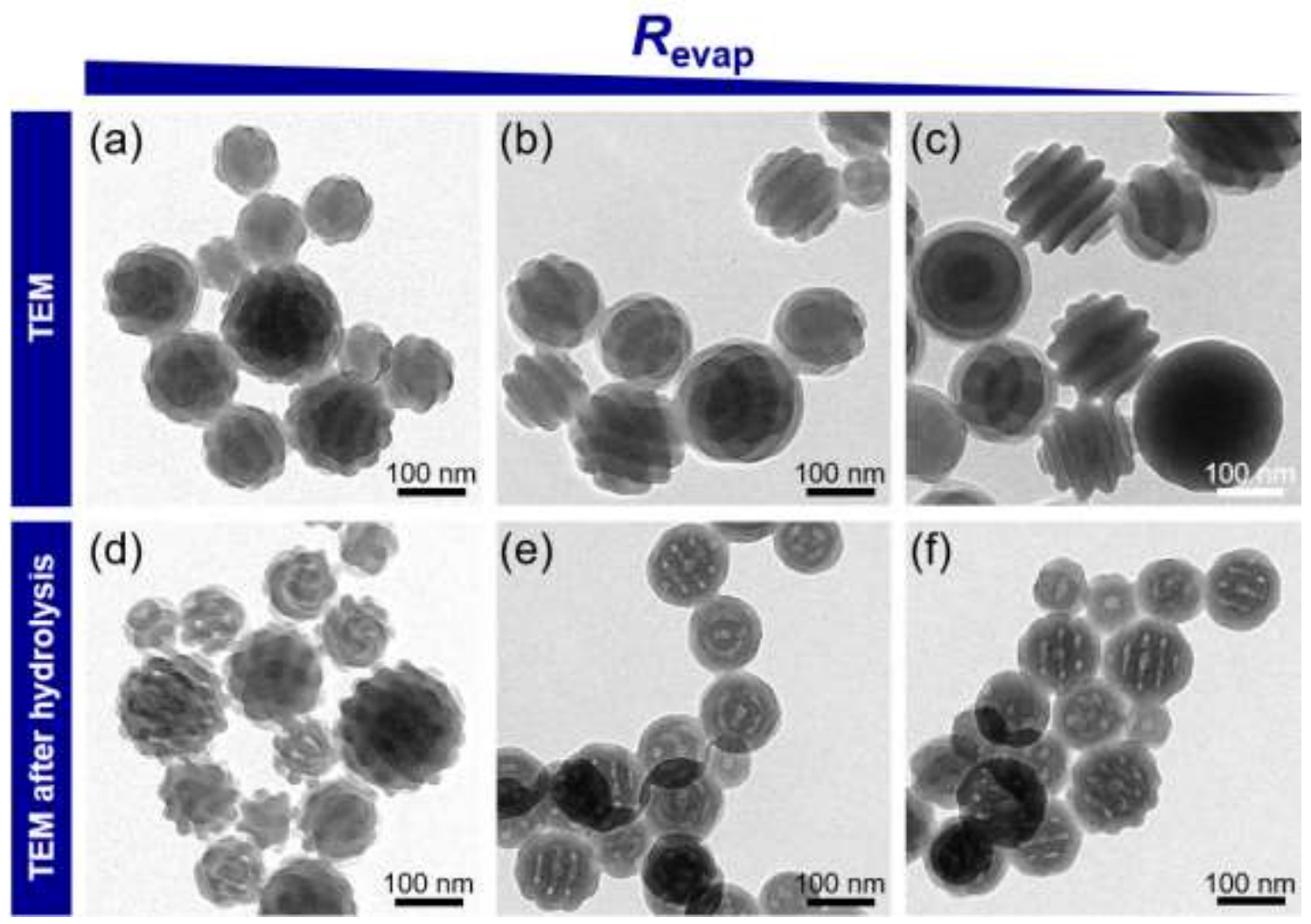

Figure S6. (a-c) TEM images of $\mathrm{PS}_{21 \mathrm{k}}-b-\mathrm{PDL} \mathrm{A}_{14 \mathrm{k}}$ particles prepared at different $R_{\text {evap values }}$ in $\mathrm{C}_{6} \mathrm{H}_{6}$ : (a) condition I, (b) condition II, and (c) condition III. (d-f) TEM images of hydrolyzed particles corresponding to (a)-(c), respectively. 


\section{Thermal annealing}

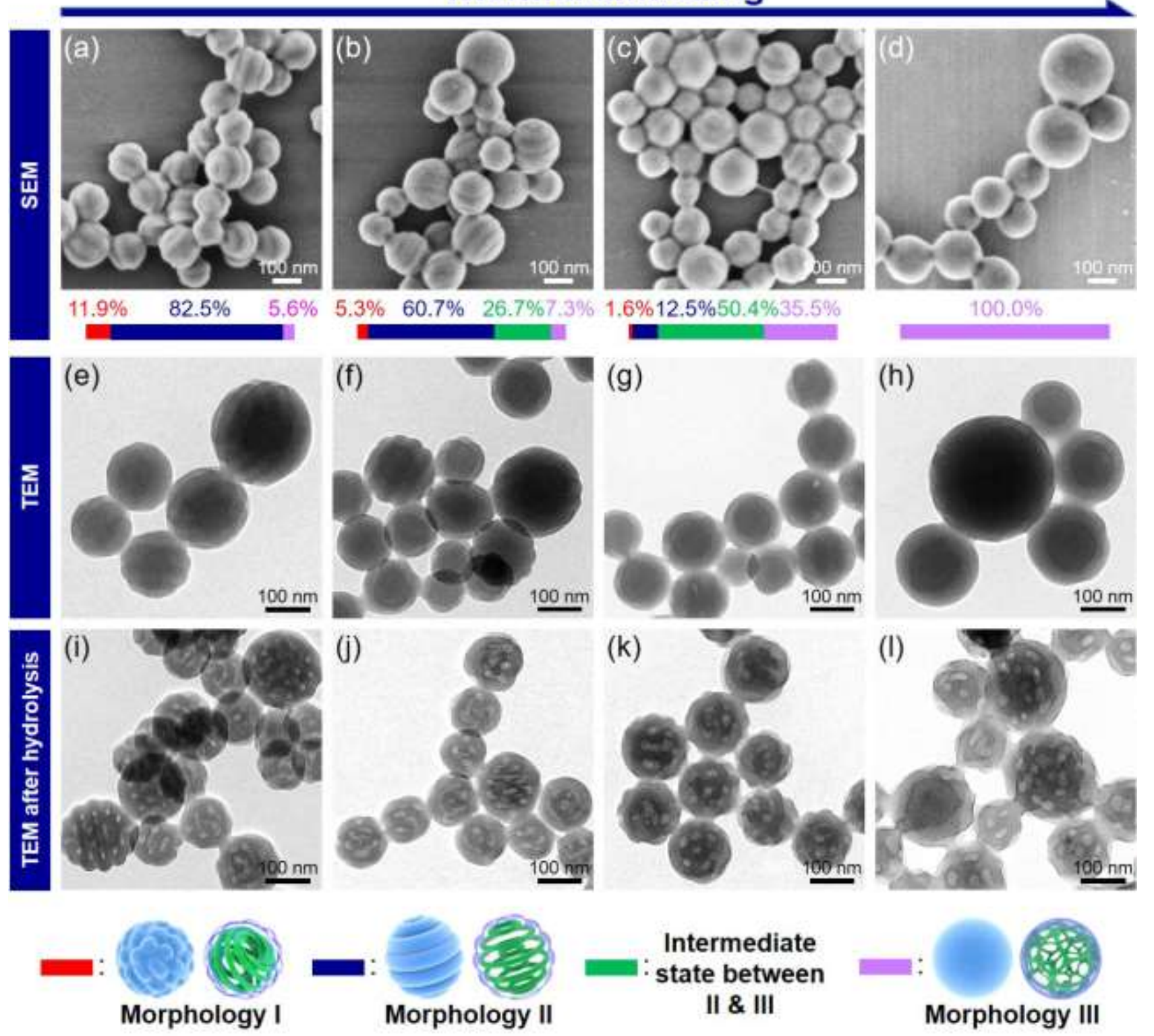

Figure S7. SEM (a-d) and TEM (e-h) images showing the topological evolution of morphology II particles under thermal annealing at $70^{\circ} \mathrm{C}$ : (a, e) $0.5 \mathrm{~h},(\mathrm{~b}, \mathrm{f}) 6 \mathrm{~h},(\mathrm{c}, \mathrm{g}) 12 \mathrm{~h}$, and (d, h) 24 h. (i-l) TEM images of hydrolyzed particles corresponding to (e)-(h), respectively, revealing the evolution of internal structure under thermal annealing. Bar charts present the frequency of each morphology, I (red), II (navy blue), an intermediate state with internal cylindrical/network structure and parallelly wrinkled surface (between II and III, olive) and III (purple), by counting 300-600 particles under each condition. 


\section{Solvent-absorption annealing}


(f)
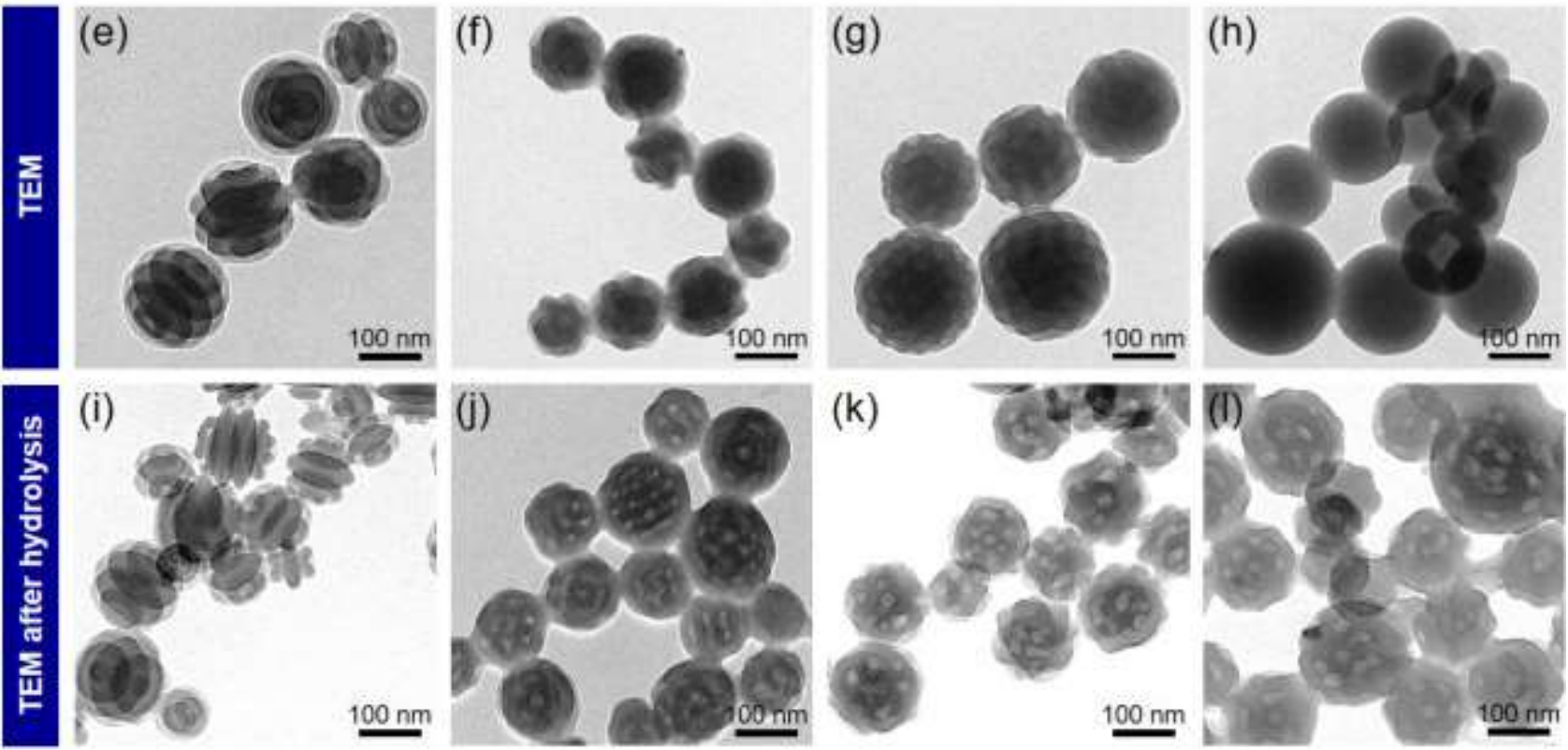

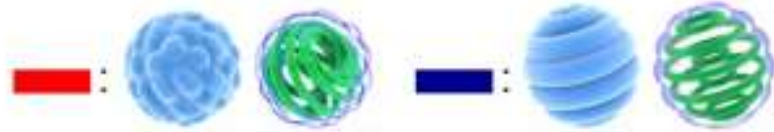

Morphology I

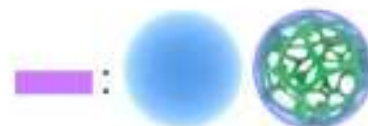

\section{Morphology III}
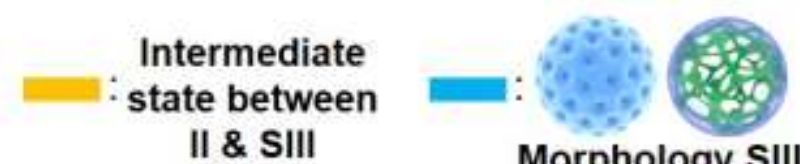

Figure S8. SEM (a-d) and TEM (e-h) images showing the topological evolution of morphology II particles under solvent-absorption annealing at $30{ }^{\circ} \mathrm{C}$ : (a, e) 1 day, (b, f) 3 days, (c, g) 7 days, and (d, h) 16 days. (i-I) TEM images of hydrolyzed particles corresponding to $(e)-(h)$, respectively, revealing the evolution of internal structure under solvent-absorption annealing. Bar charts present the frequency of each morphology, I (red), II (navy blue), an intermediate state with internal cylindrical/network structure and dotted surface pattern (between II and SIII, orange), SIII (sky blue) and III (purple), by counting 300-600 particles under each condition. 


\section{Supporting Movies:}

Movie S1. Reconstructed 3D image of hydrolyzed particles with morphology III.

Movie S2. Reconstructed cross-sectional image of hydrolyzed particles with morphology III. 